\title{
Multifaceted Convergence Study for Evaluating Gas Diffusion Parameters of Polymeric Membranes
}

\author{
Naveen Daham Weerasekera, Siyua Cao
}

\begin{abstract}
In this paper, gas transport properties of PTFE (polytetrafluorethylene) polymeric membranes are studied. We focused our work on three methods based on, Darcy's pore flow model, Fick's diffusion and numerical analysis. Effective diffusion coefficients obtained by Darcy's and Fick's laws are compared. In numerical analysis, simplified computational fluid dynamics model was created using virtual porous medium concept. Virtual porous media is generated by adding a momentum sink term in the governing equations of fluid flow. Diffusion process described by Darcy's method is performed using transfer of clean air through the membrane under induced pressure gradients. Fick's diffusion parameters are obtained based on trace compound $\left(\mathrm{C}_{3} \mathrm{H}_{8} \mathrm{O}\right)$ transport through the membrane under induced concentration gradients. Relatively high inertial permeability compared to viscous permeability $\left(\sim \mathbf{1 0}^{2}\right)$ are recorded in Darcy's analysis. Also, effective diffusion coefficient obtained by Fick's law shows $\sim 10^{-2}$ decrement compared to the same obtained by Darcy's pore flow model. From computational fluid dynamics (CFD) results, virtual pore flow model successfully demonstrated its soundness on analyzing membrane transport properties.
\end{abstract}

Index Terms - Membrane Transport, Effective Diffusion, Darcy Pore Flow Model, Fick's Diffusion, Mass Spectrometry, Virtual Porous Media, Computational Fluid Dynamics

\section{INTRODUCTION}

Polymer microporous membranes are imperative in various applications in industrial processes. Some of the prevalent applications include reverse osmosis, microfiltration, ultra-filtration, nanofiltration, gas separation, ion exchange etc. Apart from these, modern applications of nano-porous membranes extends to biotechnological processes as dialysis and targeted drug delivery [1]. The main advantage of polymeric membranes includes their wide application in sophisticated filtration devices in medical industry [2]. Furthermore, these membranes are widely used in HVAC industry to enable heat and mass transfer in recovery processes [3].

Due to their wide range of application, evaluating properties of polymeric membranes are important. Simply, a membrane can be attributed to an organized porous media with complex structure of pores having macroscopic discontinuity to continuity. Due to this complex nature of the membranes, attributing transport properties to microlevel descriptions are cumber some. As a solution to this issue, researchers introduced holistic methods to analyze transport parameters

Naveen Daham Weerasekera, Department of Mechanical and Materials Engineering, Portland State University, Portland-OR,97201, United States, +12674692310

Siyua Cao, Department of Mathematics and Statistics, Portland State University, Portland-OR,97201, United States by studying change of macroscopic variables across membranes. In brief, focusing on previous work performed by researchers on evaluating transport properties of membranes, Shoghl et al. [4] introduced a predictive gas transport model to quantify gas separation in polymer membranes. They used the solution diffusion approach extending on non-equilibrium lattice fluid (NELF) in conjunction with modified Fick's law and CFD modeling. NELF model successfully demonstrated the sorption behavior of trace gases used by Shoghl et al. [4], agreeing with experimental outcomes. Zhang et al. [3] introduced a heat and mass transfer model using energy and mass conservation equations for polymeric membrane used in energy recovery devices. From their work, they were able to plot temperature and permeability distributions in membrane surfaces based on experimental validations. Du et al. [5] studied polymer membrane for $\mathrm{CO}_{2}$ capturing processes. They reported experimental permeability characteristics of membranes also incorporating concentration isotherm approaches. Cong et al. [6] widely studied application of polymer inorganic nano-composite membranes for gas separation. They extracted the significance of membrane morphology on its transport properties. Furthermore, they also introduced application of prominent molecular level models in quantifying membrane transport properties.

Among the prevalent amount of literature available on quantifying membrane transport properties, we extended our study on the feasibility of introducing virtual porous media concept to explain and quantify these parameters. As the research gap, application of the concept of virtual porous media is not sufficiently documented. In this study, we are given an attempt to fill this gap.

It is important to have a glimpse in length scales associated with membrane transport. Since membranes are consisted of micro to nano scale pore size distributions, molecular rarefaction at some of the transport pathways becomes important. Pore channel sizes that are comparable to molecular mean free path of tracer gases are deviating from the ordinary diffusion laws, since the frequency of molecular collisions between domain walls exceed intermolecular collisions. This phenomenon is widely studied by researchers and they have proposed satisfactory models to quantify diffusion properties under this effect [7][8].

Most fundamental models that classifies membrane transport properties are Darcy's pore flow model and Fick's diffusion law. Darcy's model widely applied in porous medium where, pressure gradient across the porous domain is at importance. Contrary to this method, Fick's diffusion law observes the significance of chemical potential variation across the membrane. Furthermore, Darcy's law is able to quantify inertial and viscous permeabilities of the porous media by the help of regressive analysis. On the other hand, numerical virtual pore flow model incorporated Darcy's parameters to correlate momentum sink term in the governing equations of 
momentum transfer. The correlations are based on attributing membrane pressure drop to local fluid velocity by the help of model constants. These model constants are derived from inertial and viscous permeabilities of the membrane [9] [10]. Our current work expands on evaluating transport properties of commercially available polymer membrane by Darcy's model and Fick's diffusion laws parallel to virtual porous media concept. Our experimental rig and processes are sheltered by the work performed by Pant et al. [11] where, we integrated their experimental setup with the addition of mass spectrometry to obtain compound concentrations at the probing locations.

\section{MATERIALS AND METHODS}

\section{Semi Experimental Methods}

\section{A. Diffusivity for tracer gas in a polymeric membrane from Darcy pore flow model}

Development of the pore flow model according to Darcy-Forcheheimer law is the assumption that pressure gradient across the porous media has a variation keeping a constant concentration. Darcy's law considers the porous medium as continuous and it is satisfactory to predict a continuous flow through the porous media. In addition, Darcy's law is an expression for the Navier stokes equation after implementing certain assumptions in the continuum level and this is also called as the Darcy's scale [10]. Here the porous medium is homogeneous and isotropic, and therefore, the entrainment velocity is given by [10],

$$
v=\frac{\dot{Q}}{A}
$$

Where, $\dot{Q}$ is the volume flow rate measured and $A$ is the exposed cross-sectional area. For a circular cross section of the membrane, $A=\pi D^{2} / 4$. The popular pore flow model to describe porous media transport is the application of Darcy Forcheheimer equation. As utilized by Pant et al.[11]. Darcy's law can be given with accounting both viscous and inertial eff ects as,

$\frac{d p}{d x}=\frac{v v}{P m_{v}}+\frac{\rho v^{2}}{P m_{I}}$

Where, $P m_{v}$ and $P m_{I}$ are viscous and inertial permeabilities of the membrane for the tracer gas. And $v$ is the kinematic viscosity of the tracer gas, which can be obtained as in [11]. The pressure gradient $\frac{d p}{d x}$ is obtained as,

$\frac{d p}{d x}=\frac{P_{a}-P_{b}}{L}$

The permeability values are obtained by plotting $\frac{d p}{d x}$ against $v$. If the inertial eff ects are negligible, second term of the right-hand side take comparably very low value therefore, it can be eliminated. Therefore, the relationship between velocity vs pressure gradient can be expressed as linearly proportional to each other.

At this point necessity of introducing new diffusion parameter comes to play. Due to the complex distribution of length scales and spatially varying diffusion properties in the membrane, effective diffusion coefficient $\left(D_{\text {eff }}\right)$ is introduced. Hughes et al. [12] arrived to evaluate $D_{\text {eff }}$ by applying ideal gas laws by knowing the discrete pressure values at both sides of the membrane,

$v=\frac{D_{e f f}}{L} \ln \frac{P_{a}}{P_{b}}+\frac{P m_{v}}{v L}\left(P_{a}-P_{b}\right)$

The above equation can be attributed to molar flux $(N)$ with the use of binary friction model (BFM) where, $\mathrm{N}$ is considered as Molar flux, by Kast and Hohenthanner [13],

$\frac{d p}{d x}=R T\left(D_{e f f}+\frac{P m_{v}}{v / p}\right)^{-1} N$

Presented by Hohenthanner [13], an approximate measure for Knudsen diffusion coefficient can be obtained as,

$D_{K A}=0.89 \times \frac{1}{3} \times d \times \sqrt{\frac{8 R T}{\pi M}}$

Where, $D_{K A}$ is the Knudsen diffusivity, $d$ is the mean pore diameter, $R$ is the gas constant, $M$ is the molecular weight and $T$ is the temperature.

As more insight on effective diffusion coefficient, studies performed by Phattaranawik et al. [7] can be presented. From their approach, effective diffusion process can be represented to a parallel analogy of both Knudsen, Transition and Ordinary diffusion process as in figure 1 .

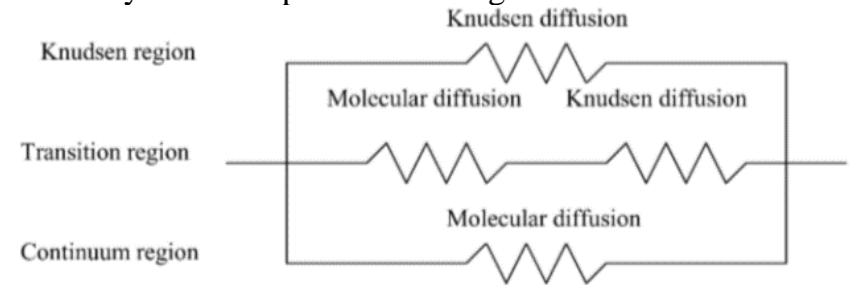

Figure 1: Representation of the analogical electrical circuit of three diffusion process

In brief, Knudsen diffusion can be defined as the diffusion process that is occurring in membrane pores when membrane pore size is comparable to molecular mean free path of the tracer gas. In this length scale, collisions between gas molecules and pore walls are dominant than intermolecular collisions. In contrary, regions that molecular diffusion occur, length scale is significantly larger than molecular mean free path. Therefore, molecular collision between domain walls can be neglected compared to intermolecular collisions. The transition region represents regions that are vulnerable to both molecular and Knudsen diffusion. In the transition region according to the pore size distribution in figure 2, the pore diameter crosses it mean pore size creating region duplicity.

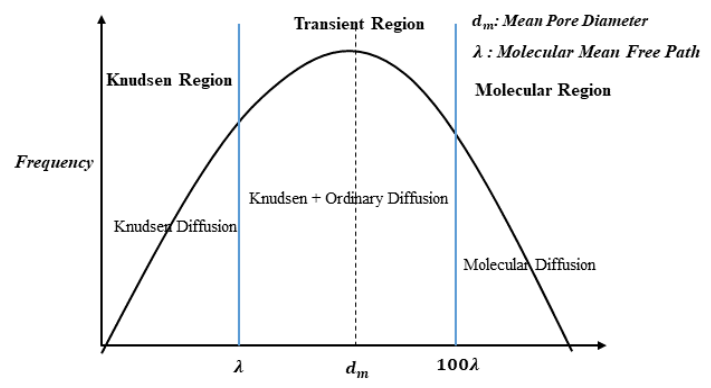

Figure 2: Regions of membrane that are vulnerable to each diffusion process.

If $D_{K A}$ is the Knudsen diffusivity and $D$ is the molecular diffusivity, then the effective diffusion coefficient can be given from the analogical electrical circuit in the transient region [7], 
$\frac{1}{D_{\text {eff }}}=\frac{1}{D}+\frac{1}{D_{k}}$

As in figure 3, Darcy's law assumed constant concentration gradient in the membrane. However, it considers a linear pressure gradient across the membrane.

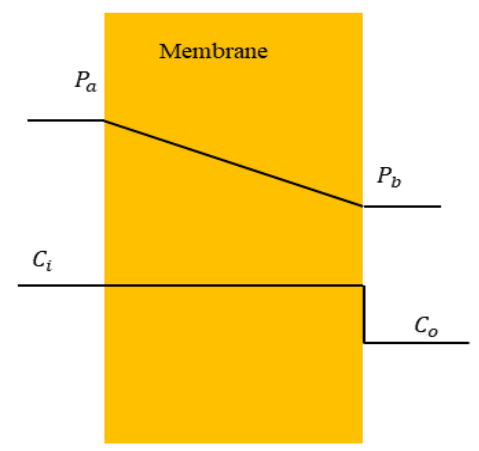

Figure 3: Assumptions in pressure and concentration in Darcy's law

Diffusivity for tracer gas in polymeric membrane from Fick's law of diffusion

When considering transport of an individual component $i$, from the Fick's law we can express the mass flux of the compound as in equation 7[9]. Here, we are defining a new effective diffusion coefficient given by Fick's law as $D_{\text {eff }-F}$, and it is applied in,

$J_{i}=D_{e f f-F} \frac{d C}{d x}=D_{e f f-F} \frac{\left(d C_{F, i}-d C_{P, i}\right)}{d x}$

Where, $C_{F, i}$ and $C_{P_{v}, i}$ are the feed and permeate side concentrations of the ith diff using component. The new eff ective diff usion coefficient, $D_{\text {eff }-F}$ solely based on the behavior of the concentration gradient and act as a lumped parameter in describing the diff usion process. All potential eff ects such as Knudsen and inertial weights are embedded inside $D_{\text {eff }-F}$, Therefore, $D_{\text {eff }-F}$ is completely evaluated experimentally. By plotting $J_{i}$ against $\frac{d C}{d x}$, for constant pressure gradients, the slope of the curve provides $D_{\text {eff }-F}$ for each average absolute pressure setting $\left(\left(P_{a}+P_{b}\right) / 2\right)$. Fick's law is based on assuming bulk concentration as the membrane-fluid interface concentration. Furthermore, this law considers constant pressure in the membrane. Therefore, the question arrives regarding the accuracy of this approach. However, the flow is transported through an externally induced pressure gradient which provides continuous molecular interactions between surface layer and bulk fluid, satisfactorily reduce above error according to Wijman [9]. The compound flux $\mathrm{J}_{\mathrm{i}}$ is calculated from the measurements of exit concentration $C_{P_{s} O}$ and inlet concentration $C_{P_{s} i}$ as,

$$
J_{i}=\frac{\dot{Q} \times\left(C_{P, i}-C_{P, o}\right)}{A}
$$

\section{Experimental Setup}

Experimental test rig is fabricated to monitor pressure difference, volume flow rate and compound concentrations across the membrane. We used a commercial PTFE membrane with $0.2 \mu \mathrm{m}$ mean flow diameter with standard deviation of $0.01 \mu \mathrm{m}$. Membrane was placed between two inlet and exhaust channels (Polystyrene ducts) with rubber gaskets to prevent leakages from the interfaces as in figure 5 . Four measurement taps are created in each channel to monitor volume flow rate, concentration and pressure. Pressure difference is monitored by pressure transducer (Omega Instruments-PX2300-10DI) connected to taps in inlet and exhaust channels. Entrained flow is directed towards the Gilibrator (Sensidyne Gilibrator Version-2). Gilibrator is capable of measuring with a minimum of $1 \mathrm{~cm}^{3} / \mathrm{min}$. Inlet channel is tapped to a pressurized fresh air supply stream through a control valve. Trace compound is supplied by using a customarily fabricated VOC generator and it is allowed to mix with incoming purified air by a use of T-joint. Air is purified with three process. First, it is sent through granular filter (Thomas Scientific) that is capable of removing micron level particulate matter. Secondly, it is directed through an activated carbon filter (Thomas Scientific-HEPA) where any microbial level contaminants are removed. Finally, through the deaerator (Thomas Scientific) to remove moisture. Membrane assembly and channel arrangements are strictly tested for leakages. This is tested with the use of ultrafine smoke pencil (Specifications) probing around the vulnerable areas. However, undetectable leakages do not impact the overall experimental outcomes, since, the calculations are based on differences in pressure and differences in concentration. However, these leakages are contributing to minor error in volume flow measurements.

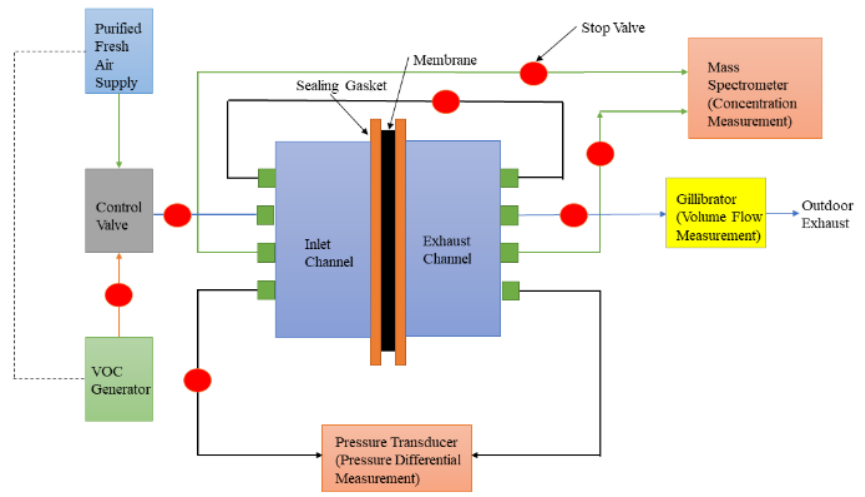

Figure 5: Experimental Setup

Figure 6 presents the fabricated test rig. All tubing is done through laboratory standard Teflon materials where, VOC emissions from tube walls are minimized. We used isopropyl alcohol as the trace compound for all experiments due its availability in pure form and high solubility in water. Mass spectrometer used is proton transfer reaction time of flight (PTR-TOF) type (Ionicon Analytik-800). Initially mass spectrometer is calibrated for acetone as per the standard process presented by Laguerre et al. [14].

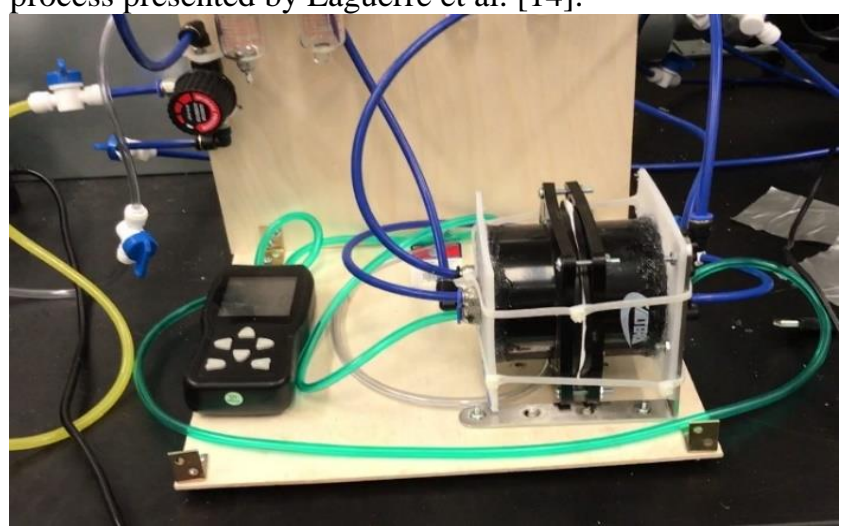

Figure 6: Picture of the fabricated test rig 


\section{B. Experimental Protocol}

Experiments are performed to obtain variation of pressure gradient and concentration gradient with respect to velocity of flow across the membrane. For the phase 1, Initially, an air supply is increased until Gilibrator responds. After Gilibrator response has been obtained, fresh air supply is incremented with $10 \mathrm{~cm}^{3}$ intervals and respective pressure differences are measured. This procedure is repeated multiple times until convergence of measurements achieved. As per the phase 2, volume flow rate across the membrane kept constant. However, in this process, trace compound is supplied by the VOC generator to the supply air stream. Mass spectrometric ports are opened in repeated manner by inlet and exhaust taps to measure concentrations. It is important to note that due to mass spectrometric sampling, pressure loss occurs at either side of the membrane $(20 \mathrm{~Pa})$, since mass spectrometer is utilizing a vacuum pump. However, loss in pressure is equal in both sides of the membrane and since experiments are conducted at constant pressure, negative effects from this issue are avoided.

Table 1: Experimental protocol and its significance.

\begin{tabular}{|l|l|l|}
\hline $\begin{array}{l}\text { Experime } \\
\text { ntal Phase }\end{array}$ & Protocol & Application \\
\hline Phase 1 & $\begin{array}{l}\text { No trace compound is } \\
\text { injected. } \\
\text { Airflow rate across } \\
\text { the membrane is } \\
\text { varied. } \\
\text { Pressure difference is } \\
\text { measured } \\
\text { respectively. }\end{array}$ & $\begin{array}{l}\text { Obaining effective } \\
\text { represented by } \\
\text { Darcy's law. }\end{array}$ \\
\hline Phase 2 & $\begin{array}{l}\text { Constant volume flow } \\
\text { rate across the } \\
\text { membrane. } \\
\text { Trace compound is } \\
\text { injected. } \\
\text { Concentration of the } \\
\text { trace compound is } \\
\text { varied at the inlet of } \\
\text { the membrane. } \\
\text { Individual } \\
\text { concentration at the } \\
\text { inlet and exit of the } \\
\text { membrane } \\
\text { measured. }\end{array}$ & $\begin{array}{l}\text { Fick's } \\
\text { represented } \\
\text { constant pressure. }\end{array}$ \\
\hline
\end{tabular}

Isopropyl alcohol $\left(\mathrm{C}_{3} \mathrm{H}_{8} \mathrm{O}\right)$ is used as the trace compound, which is a volatile organic compound (VOC). Isopropyl alcohol is used since its availability in pure form and its high-water solubility. High-water solubility allows to produce custom concentrations by producing better interphase mass transfer using bubbling process. Also, from previous literature [14] there is good transfer properties recorded for high water-soluble compounds in polymeric membranes that are in detectable range.

\section{Computational Fluid Dynamics with Virtual Porous Media}

To model a porous media by accounting all the microscopic structures is burdensome and also impossible for a random complex geometry. Therefore, a modification for the general Navier-Stokes (NS) equations are introduced through an additional source term representing the porous media resistance [15]. In practice, this source term produces an additional pressure drop apart from the viscous pressure drop due to the presence of porous media.

$$
\frac{\partial \rho v}{\partial t}=\nabla \cdot(\rho v \cdot v)=-\nabla \cdot p+\nabla \cdot \tau-F
$$

Where, $F$ is the additional sink term added and it is represented by Darcy-Forcheimer equations. $F$ is modeled as,

$$
F_{i}=\frac{\partial p}{\partial x}=-\frac{\mu}{\alpha} \cdot v_{i}+0.5 \cdot C_{2} \rho[v] v_{i}
$$

Where, $\alpha$ is the viscous permeability and $1 / C_{2}$ is the inertial permeability. Inverse of these parameters represents the viscous and inertial resistances respectively for the porous media. In this step, through the available experimental data for pressure diff erences and entrained volume flow rates, the inertial and viscous resistances are calculated and fitted to the most probabilistic curve by simulating flow itself for arbitrary values. For CFD approaches, equation 10 is simplified to following form as a corollary from equation 2 [15],

$$
\Delta p=a \cdot v_{i}^{2}+b \cdot v_{i}
$$

By comparing this representation with the original equation 2, inertial and viscous resistances are obtained as follows,

$$
\begin{aligned}
& \frac{1}{\alpha}=\frac{b}{\mu \cdot \Delta x_{i}} \\
& C_{2}=\frac{2 a}{\rho \Delta x_{i}}
\end{aligned}
$$

Where, $\Delta x_{i}$ is the thickness of the porous material in $i^{\text {th }}$ direction.

\section{Computational Model and Grid Independence}

CFD model is generated using ANSYS Fluent 2019 software in 2D. Generating a model with length scales equal to experimental setup creates computational difficulties. Since the thickness of the membrane $(\mathrm{L})$ is $0.5 \mathrm{~mm}$ and its diameter (D) is $90 \mathrm{~mm}, \mathrm{D} / \mathrm{L}$ ratio becomes $180>>10$. This produces an unavoidable issue in meshing because sufficient resolution of the mesh at membrane is unable to obtain. Therefore, as a solution, a microscale model is generated emphasizing local transport phenomena at the membrane. Figure 5 represents the basics of the CFD model.

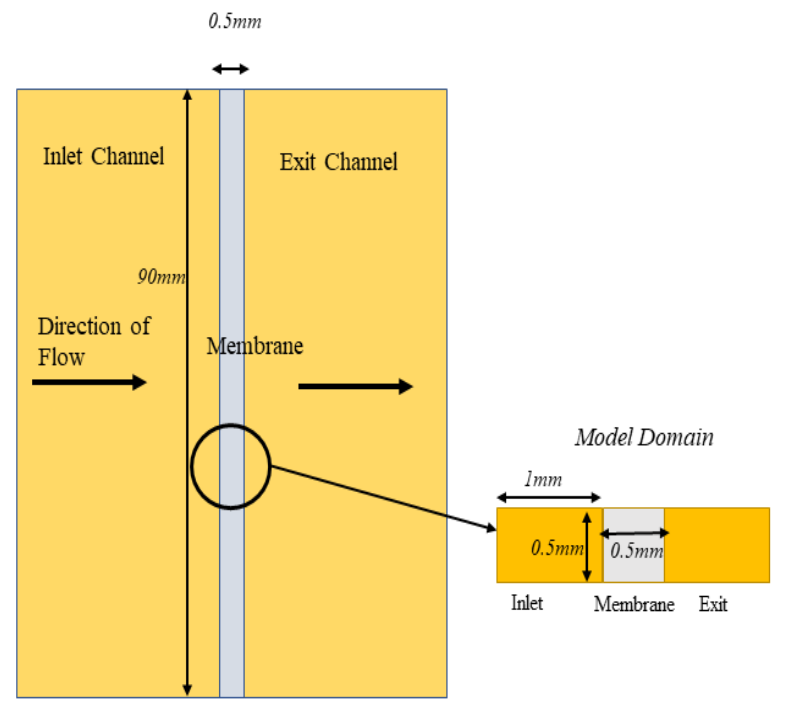

Figure 5: Development of the CFD model. 
As in figure 5, model geometry is created with $0.5 \mathrm{~mm} \times 0.5 \mathrm{~mm}$ porous domain with two $1 \mathrm{~mm} \times 0.5 \mathrm{~mm}$ flow channels. Membrane properties are applied to the porous domain defining values of inertial and viscous resistances as represented by equations 12 and 13. Mesh is generated and finalized based on grid independence. When developing the model, we assumed that pressure variation far away from the membrane is uniform and it does not impact local results. In other words, it is assumed that entire pressure difference between channel entry and exit of the experimental setup is represented by the pressure difference between inlet and exit boundaries of the model. Generated mesh is depicted in figure 6.

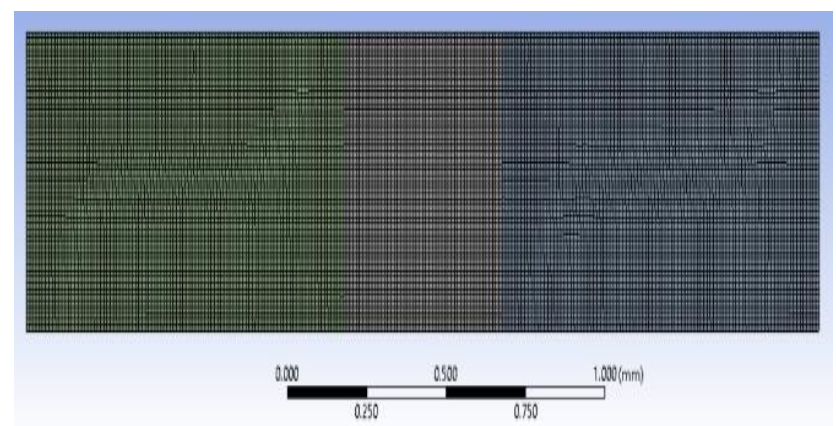

Figure 6: Mesh of the domain

Analog to measurements, boundary conditions are applied from the basis of pressure differences. Gauge pressure at the channel inlet is varied according to measurements and gauge pressure at the channel exit was set to zero representing ambient pressure. Parameter of the porous media are changed to fit the simulation results that to the experimental data. The correlation is carried with respect to velocity across the channel with pressure gradient. This approach is applicable since the experimental velocity across a unit cross section is equal to the average velocity. Therefore, holistic velocity equals to the local velocity at a minor cross section of the membrane. For turbulence modeling, k- $\omega$ model with Mentor's shear stress transport (SST) model is applied. This model is well documented for its successfulness in operation under adverse pressure gradients [16]. Because porous region is a region which is highly vulnerable to adverse pressure gradients, this action is taken.

\section{RESULTS AND DISCUSSION}

\section{Experimental Outcomes}

\section{A. Phase 1}

The experimental outcomes from phase 1 are shown in figure 7. There is almost linear variation between pressure difference and velocity across the membrane. Relatively low velocities across the membrane are also observed $\left(\sim 10^{-5}\right)$. At very low-pressure difference, after extending the graphs until it intersects $y$ axis, the intercept almost reaches zero producing very low values for viscous and inertial permeabilities $\left(\sim 10^{-15}\right)$. These values are congruent with the values reported by previous literature [11]. Furthermore, the effective diffusion coefficient obtained using Darcy's method creates a greater variation with the effective diffusion coefficient obtained by the BFM as in figure 7-(d).
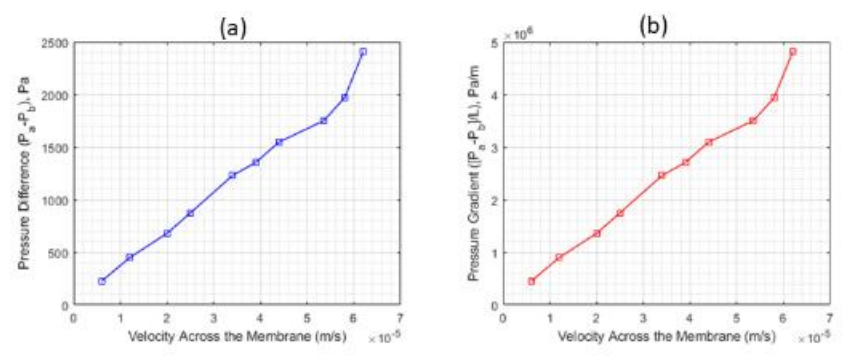

(c)
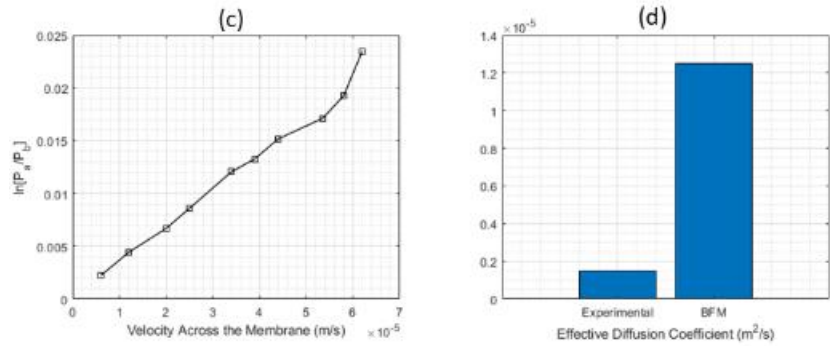

Figure 7: (a)-Variation pressure difference with velocity across the membrane, (b)-variation of pressure gradient with

velocity, (c)-Variation of $\ln \left(\mathrm{P}_{\mathrm{a}} / \mathrm{P}_{\mathrm{b}}\right)$ with velocity, (d)-

Comparison between experimental and model diffusion coefficients

Table 2: Summary of the diffusion parameters obtained by Darcy's analysis $(0.45 \mu \mathrm{m}$ mean pore diameter)

\begin{tabular}{|c|c|c|c|}
\hline Viscous & Inertial & Experimental & Effective \\
$P m_{v},\left(\mathrm{~m}^{2}\right)$ & $P m_{I},(\mathrm{~m})$ & $\begin{array}{c}\text { Effective } \\
D_{\text {eff }},\left(\mathrm{m}^{2} / \mathrm{s}\right)\end{array}$ & $\begin{array}{c}\text { Diffusivity } \\
\text { from BFM, } \\
\left(\mathrm{m}^{2} / \mathrm{s}\right)\end{array}$ \\
& & & \\
\hline $2.0155 \times 10^{-16}$ & $5.23 \times 10^{-14}$ & $1.488 \times 10^{-6}$ & $1.2502 \times 10^{-5}$ \\
\hline
\end{tabular}

B. Phase 2

In this process, the experiments are started with an initial concentration of $2.5 \mathrm{mg} / \mathrm{m}^{3}$ of the trace compound at the membrane inlet. The pressure difference is set to $1350 \mathrm{~Pa}$ throughout. Concentration at the inlet in incremented approximately by $1 \mathrm{mg} / \mathrm{m}^{3}$ and respective outlet concentration is measured. Figure 8 shows experimental outcomes from phase 2 .
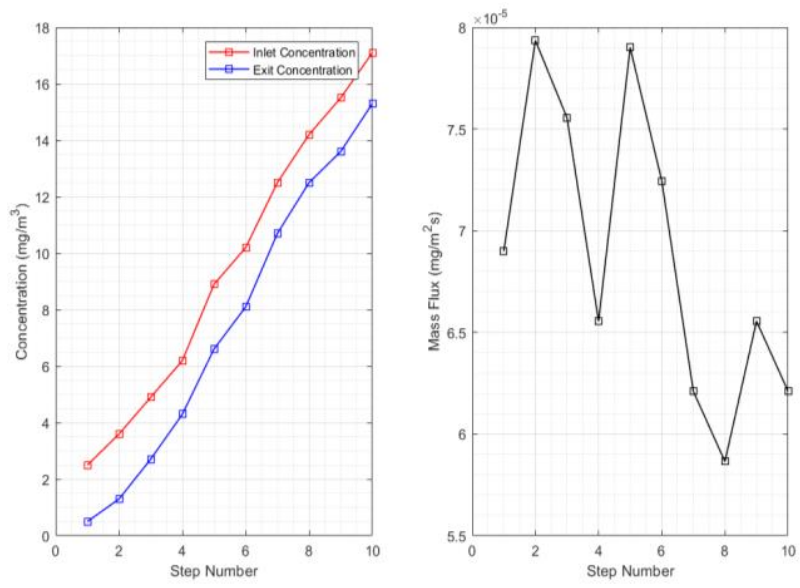

Figure 8: Concentration and mass flux variation, (left)- Inlet and exit concentration of the membrane, (right)- Mass flux across the membrane (Diffusion Coefficient:

$$
\left.1.725 \times 10^{-8} \mathrm{~m}^{2} / \mathrm{s}\right)
$$


From figure 7, it is observable that outlet concentration almost follows the inlet concentration pattern with minor deviations. Mass flux varies with the concentration difference $\left(\mathrm{C}_{\mathrm{in}}-\mathrm{C}_{\text {out }}\right)$ with a mean of $6.67 \mathrm{mg} / \mathrm{m}^{2} \mathrm{~s}$. By curve fitting above data with equation 7, Fick's diffusion coefficient is obtained as, $1.725 \times 10^{-8} \mathrm{~m}^{2} / \mathrm{s}$. Comparing the value of Fick's diffusion coefficient and Darcy's diffusion coefficient, there is an order of magnitude difference of $\sim 10^{-2}$ can be observed.

Table 3: Summary of parameters obtained by Fick's law analysis

\begin{tabular}{|c|c|}
\hline $\begin{array}{c}\text { Mean Mass Flux Across the } \\
\text { Membrane }\left(J_{i}\right) \\
\left(\mathrm{mg} / \mathrm{m}^{2} \mathrm{~s}\right)\end{array}$ & $\begin{array}{c}\text { Effective Diffusion } \\
\text { Coefficient }\left(D_{\text {eff }}-F\right)\left(\mathrm{m}^{2} / \mathrm{s}\right)\end{array}$ \\
\hline 6.67 & $1.725 \times 10^{-8}$ \\
\hline
\end{tabular}

\section{CFD Outcomes}

In CFD simulations, it is observed that with applied pressure differences according to phase, 1 at the inlet and exit boundary conditions in the model, relatively similar velocity patterns are observed except for the magnitude. Figure 9 presents the contour plots obtained for applied pressure difference of $642 \mathrm{~Pa}$. It is important to note that parameters obtained from phase 1 (inertial and viscous resistances), are applied to porous media properties in the model.

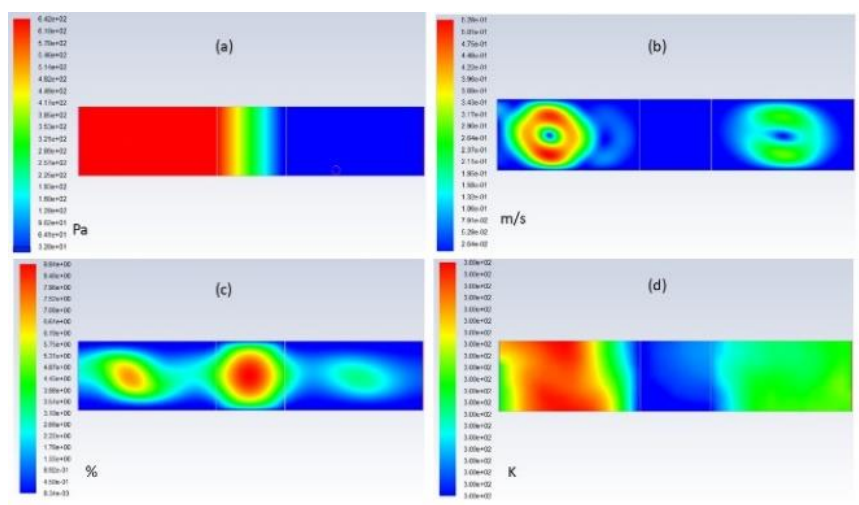

Figure 9: Typical simulation result obtained for $642 \mathrm{~Pa}$ pressure difference across the membrane, (a)-Contours of gauge pressure $(\mathrm{Pa}),(\mathrm{b})$-Contours of velocity $(\mathrm{m} / \mathrm{s})$,

(c)-Contours of turbulence (\%) intensity, (d)-Contours of temperature $(\mathrm{K})$

As in the real case, rapid pressure drop is observed at the membrane due to high flow resistance offered by the membrane. Also, high relative magnitude of velocity is observed at the middle of the inlet channel. This is occurring due to the backflow by the impingement of flow on the membrane surface. Parallel to inlet channel, at the exit channel, a circulatory velocity profile is observed. This is occurring due to the spraying effect of air from the membrane pores to the exit channel. Although relatively very low velocities are observed in the membrane, high turbulent intensities are recorded. This is an approximate representation of the turbulent flow in pore channels, which is not exact. However, virtual pore flow model is able to express the turbulence at the porous media for some extent. Figure 10 represents the variation of fluxes across the membrane.
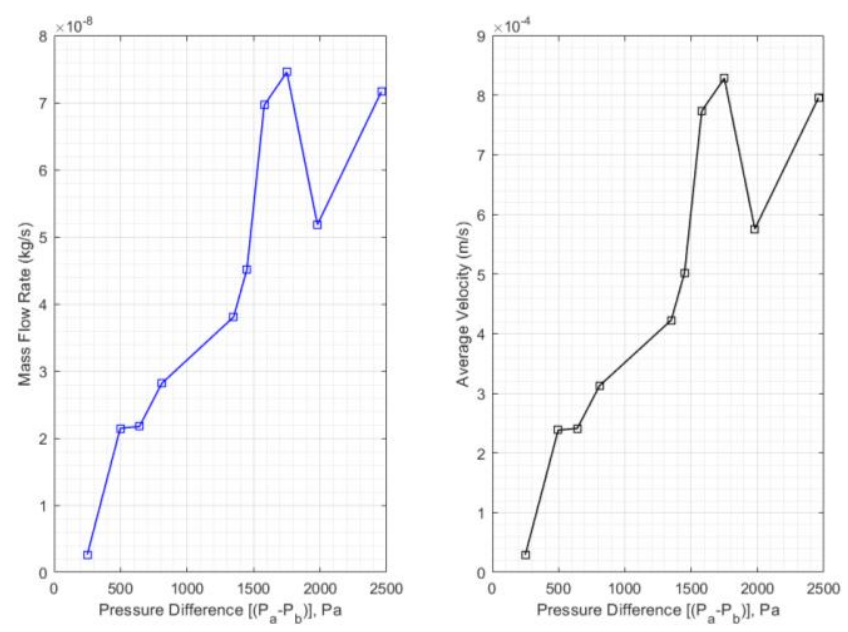

Figure 10: Computed fluxes across the membrane, (left)-

Variation of mass flow rate across the membrane with pressure difference, (right)- Variation of average velocity with pressure difference

As in figure 10, mass flux is computed at the channel exit (Exit boundary face). Average velocity is computed assuming considered model region is located at the middle of the experimental membrane. Therefore, velocity is derived based on channel of width $1 \mathrm{~mm}$ and length of $90 \mathrm{~mm}$. Comparing average velocity obtained from the model with experimental velocity across the membrane, a difference in order of magnitude of $\sim 10^{-1}$ is observed. This is a satisfactory deviation from the experimental values, since velocities near the membrane are higher than that of far away from the membrane-fluid interface, contrary to the velocities measured in phase 1. Furthermore, there is a rapid drop-in mass flow rate was observed at high pressure difference of $\sim 2000 \mathrm{~Pa}$. We predict this effect is observable due to high turbulence and backflow present at the inlet channel at high pressure gradients.

\section{CONCLUSION}

In this paper, a multifaceted study is performed on evaluating gas transport properties of polymeric membranes. Three distinct studies are performed on the basis of experimental and numerical approaches. In the first study, diffusion parameters were obtained by Darcy's pore flow model are observed. Secondly, Fick's diffusion law is studied focusing on transport of a trace organic compound through the polymeric membrane. Thirdly, a numerical simulation is performed by creating a membrane model attributed to virtual porous media. The porous media is modeled by introducing a sink term in the momentum equation at the porous domain. Highest effective diffusion coefficient is obtained by Darcy's pore flow model averaged to $1.488 \times 10^{-6} \mathrm{~m}^{2} / \mathrm{s}$, which is $10^{-1}$ order of magnitude lower than the diffusion coefficient predicted by BFM $\left(1.2502 \times 10^{-5} \mathrm{~m}^{2} / \mathrm{s}\right)$. Lowest, effective diffusion coefficient is obtained in the analysis confirming Fick's diffusion of $1.725 \times 10^{-8} \mathrm{~m}^{2} / \mathrm{s}$. Furthermore, CFD simulations confirmed the applicability of current virtual pore flow model to calibrate membrane properties. Also, from the analysis, it is evident that advection induced transport is dominant than molecular diffusion induced transport in polymeric membranes with a mean pore diameter in the range of $0.2-0.8 \mu \mathrm{m}$. 


\section{ACKNOWLEDGMENT}

Naveen Daham Weerasekera with Siyua Cao wish to thank Dr. Elliott Gall at the Department of Mechanical and Materials Engineering of Portland State University and Dr. Bin Jiang at the Department of Mathematics and Statistics of Portland State University, USA for their constant support throughout this work.

This work is performed by the startup funds provided by the Department of Mechanical and Materials Engineering of Portland State University, Portland, OR-97201, USA.

\section{REFERENCES}

[1] Adam Mendelsohn Tejal Desai, "Inorganic Nanoporous Membranes for Immunoisolated Cell-Based Drug Delivery", "Advances in Experimental Medical Biology", 2010, 670: 104-125

[2] Dunleavy M, "Polymeric membranes. A review of applications", "Medical Device Technology", 1996, 7(4), 18-21

[3] L.Z. Zhang, Y. Jiang, "Heat and mass transfer in a membrane-based energy recovery ventilator", "Journal of Membrane Science”, 163 (1999) 29-38

[4] Sina Nabati Shoghl, Ahmadreza Raisi, Abdolreza Aroujalian, "A predictive mass transport model for gas separation using glassy polymer membranes", "Royal Society of Chemistry", 2015, 5, 38223-38234

[5] Naiying Du, Ho Bum, Park,GillesP.Robertson, Mauro M. Dal Cin,TymenVisser, Ludmila Scoles, Michael D. Guiver, "Polymer nanosieve membranes for $\mathrm{CO} 2$-capture applications", "Nature Materials", 2011, Vol.10

[6] Hailin Cong, Maciej Radosz, Brian Francis Towler, Youqing Shin, "Polymer inorganic nano-composite membranes for gas separation", "Separation and Purification Technology", 55(2007), 281-291

[7] J. Phattaranawik, R. Jiraratananon, A.G. Fane, "Effect of pore size distribution and air flux on mass transport in direct contact membrane distillation", "Journal of Membrane Science" 215 (2003) 75-85

[8] J A M Kerkhof, "A modified Maxwell-Stefan model for transport through inert membranes: The binary friction model", "Chemical Engineering Journal and Biochemical Engineering Journal", 64:319-343, 1996.

[9] R W Baker J G Wijmans, "The solution diff usion model: A review", "Journal of Membrane Science", 107:1-21, 1995.

[10] Stephen Whitaker, "Flow in porous media: Theoretical derivation of Darcy's law", "Transport in Porous Media", 3:25, 1985.

[11] Lalit M Pant, Sushanta K Mitra, Marc Secanell, "Absolute permeability and Knudsen diff usivity measurements in PEMFC gas diff usion layers and microporous layers", "Journal of Power Sources", 206:153-160, 2012.

[12] K Hughes, D Ingham L., Ma M Pourkahsa, M Rosil, M Ismail, T. Damjanovic. “Journal of Fuel Cell Technology”, 7:161-167, 2010.

[13] C R Hohenthanner,W Kast, "Tortuisity of electrochemical devices" "International Journal of Heat and Mass Transfer", 43:319-343, 2000.

[14] A. Laguerre, P. Ramasubramanian, M. Survilo, M. Duenas, N. D. Weerasekera, L. A. George, E. T. Gall, "Indoor and Outdoor Levels of Traffic-Related Air Pollution (TRAP) and Effectiveness of Remediation Measures in a Near Freeway School", "The American Association for Aerosol Research (AAAR) $37^{\text {th }}$ Annual Conference (2019)", Portland, OR.

[15] Remigiusz Nowak, " Estimation of viscous and inertial resistance coefficients for various heat sink configurations", "Proceedia Engineering", 157, 2016, 126-131

[16] Mentor, F. R. (August 1994). "Two-Equation Eddy-Viscosity Turbulence Models for Engineering Applications". "AIAA Journal." 32 (8): 1598-1605. Bibcode:1994AIAAJ..32.1598M. doi:10.2514/3.12149

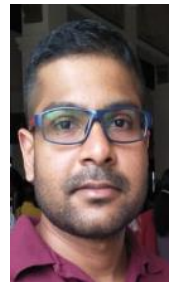

Naveen Daham Weerasekera is a Former Graduate Research Assistant at the Department of Mechanical and Materials Engineering, Portland State University, USA $\mathrm{He}$ is currently a doctoral candidate in Mechanical Engineering. His research interests expand on microfluidics, nano-scale transport properties, mass spectrometry and particle dynamics. Currently his research is funded by STARR research grant awarded by the Portland State Institute for Sustainable Solutions

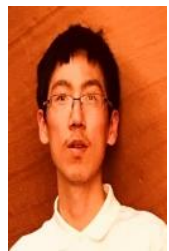

Siyua Cao is a Graduate Research Assistant at the Department of Mathematics and Statistics, Portland State University, USA. He obtained his master's and bachelor's degrees in mathematics from Portland State University. $\mathrm{He}$ is an active researcher in numerical methods and complex boundary value problems on solving governing equations related to scaler transport. 